\title{
A variational approach of Sturm-Liouville problems with the nonlinearity depending on the derivative
}

\author{
Ghasem A Afrouzi' , Armin Hadjian² and Vicenţiu D Rădulescu 3,4*
}

\author{
"Correspondence: \\ vicentiu.radulescu@imar.ro \\ ${ }^{3}$ Institute of Mathematics 'Simion \\ Stoilow' of the Romanian Academy, \\ P.O. Box 1-764, Bucharest, 014700, \\ Romania \\ ${ }^{4}$ Topology, Geometry and Nonlinear \\ Analysis Group, Department of \\ Mathematics, University of \\ Ljubljana, Jadranska 21, SI-1000, \\ Ljubljana, Slovenia \\ Full list of author information is \\ available at the end of the article
}

\begin{abstract}
In this paper, we are concerned with the existence of positive classical solutions for a class of second-order differential equations with the nonlinearity dependent on the derivative. We also provide a range of the parameter in order to obtain the existence of multiple solutions. The approach is based on variational methods. An example illustrates the abstract results of this paper.
\end{abstract}

MSC: 34B15; 35B38; 58E05

Keywords: Dirichlet problem; critical points; variational methods

\section{Introduction}

In 1836 to 1837, the French mathematicians Jacques Charles François Sturm (1803-1855) and Joseph Liouville (1809-1882) published several papers that initiated a new subtopic of mathematical analysis: the Sturm-Liouville theory. Sturm and Liouville were concerned with the general linear homogeneous second-order differential equation of the form

$$
\left(p(x) u^{\prime}\right)^{\prime}+q(x) u=\lambda w(x) u \quad \text { if } x \in[a, b]
$$

where the potentials are given functions. Under various boundary conditions, Sturm and Liouville established that solutions of problem (1.1) can exist only for particular values of the real parameter $\lambda$, which is called an eigenvalue. Relevant examples of linear SturmLiouville problems are the Bessel equation and the Legendre equation.

The classical Sturm-Liouville theory does not depend upon the calculus of variations but stems from the theory of ordinary linear or nonlinear differential equations. Linear SturmLiouville equations can be also studied in the context of functional analysis by means of self-adjoint operators or integral operators with a continuous symmetric kernel (the Green's function of the problem). Certain applications involving linear partial differential equations can be treated with the help of the Sturm-Liouville theory, for instance the normal modes of vibration of a thin membrane. We also refer to [1], where a perturbed nonlinear Sturm-Liouville problem with superlinear convex nonlinearity is studied. In the recent paper [2], the authors study a class of discrete anisotropic Sturm-Liouville problems. We also refer to [3-5] for related properties of solutions of Sturm-Liouville problems.

(c) 2015 Afrouzi et al.. This article is distributed under the terms of the Creative Commons Attribution 4.0 International License (http://creativecommons.org/licenses/by/4.0/), which permits unrestricted use, distribution, and reproduction in any medium, provided you give appropriate credit to the original author(s) and the source, provide a link to the Creative Commons license, and indicate if changes were made. 
In the present paper, we are concerned with a class of nonlinear Sturm-Liouville problems and we establish some qualitative properties of the eigenvalues by using variational principles. A feature of our work is the presence of the derivative in the nonlinear term, which creates further technical constraints.

Our main purpose in this paper is to establish a range of eigenvalues in a suitable interval in order to create at least one eigenfunction. As a consequence, we establish sufficient conditions for the existence of two or three solutions.

Consider the following quasilinear Sturm-Liouville problem with Dirichlet boundary condition on a bounded interval $[a, b]$ in $\mathbb{R}$ :

$$
\left\{\begin{array}{l}
-(p-1)\left|u^{\prime}(x)\right|^{p-2} u^{\prime \prime}(x)=\lambda f(x, u) h\left(x, u^{\prime}\right), \quad x \in(a, b) \\
u(a)=u(b)=0
\end{array}\right.
$$

We assume that $p>1, \lambda$ is a positive parameter, $h:[a, b] \times \mathbb{R} \rightarrow[0,+\infty)$ is a bounded and continuous function with $m:=\inf _{(x, t) \in[a, b] \times \mathbb{R}} h(x, t)>0$ and $f:[a, b] \times \mathbb{R} \rightarrow \mathbb{R}$ is an $L^{1}$-Carathéodory function.

Dirichlet boundary value problems have been widely studied because of their applications to various fields of applied sciences such as mechanical engineering, control systems, computer science, economics, artificial or biological neural networks and many others.

In this connection, several existence and multiplicity results for solutions to secondorder ordinary differential nonlinear equations, with the nonlinearity dependent on the derivative and Dirichlet conditions at the ends, have been investigated making use of variational methods.

For instance, Graef et al. in [6], by using a three critical point theorem of Ricceri [7], proved the existence of at least three classical solutions for the Dirichlet quasilinear elliptic system

$$
\left\{\begin{array}{l}
-\left(p_{i}-1\right)\left|u_{i}^{\prime}(x)\right|^{p_{i}-2} u_{i}^{\prime \prime}(x) \\
\quad=\left[\lambda F_{u_{i}}\left(x, u_{1}, \ldots, u_{n}\right)+\mu G_{u_{i}}\left(x, u_{1}, \ldots, u_{n}\right)\right] h_{i}\left(x, u_{i}^{\prime}\right), \quad x \in(a, b), \\
u_{i}(a)=u_{i}(b)=0 \quad \text { for } 1 \leq i \leq n
\end{array}\right.
$$

where $p_{i}>1$ for $1 \leq i \leq n, \lambda>0$ and $\mu \geq 0$ are parameters, $a, b \in \mathbb{R}$ with $a<b, h_{i}:[a, b] \times$ $\mathbb{R} \rightarrow[0,+\infty)$ is a bounded and continuous function with $m_{i}:=\inf _{(x, t) \in[a, b] \times \mathbb{R}} h_{i}(x, t)>0$ for $1 \leq i \leq n, F:[a, b] \times \mathbb{R}^{n} \rightarrow \mathbb{R}$ is a function such that the mapping $\left(t_{1}, t_{2}, \ldots, t_{n}\right) \rightarrow$ $F\left(x, t_{1}, t_{2}, \ldots, t_{n}\right)$ is in $C^{1}$ in $\mathbb{R}^{n}$ for all $x \in[a, b], F_{t_{i}}$ is continuous in $[a, b] \times \mathbb{R}^{n}$ for $1 \leq$ $i \leq n$, and $F(x, 0, \ldots, 0)=0$ for all $x \in[a, b]$. Here, $F_{t_{i}}$ denotes the partial derivative of $F$ with respect to $t_{i}$. Similarly, $G:[a, b] \times \mathbb{R}^{n} \rightarrow \mathbb{R}$ is a function such that $\left(t_{1}, t_{2}, \ldots, t_{n}\right) \rightarrow$ $G\left(x, t_{1}, t_{2}, \ldots, t_{n}\right)$ is in $C^{1}$ in $\mathbb{R}^{n}$ for all $x \in[a, b]$, and $G_{t_{i}}$ is continuous in $[a, b] \times \mathbb{R}^{n}$ for $1 \leq i \leq n$.

Successively, in [8], the authors considered the system (1.3) in the case $\mu=0$, finding the existence of infinitely many classical solutions for certain values of the parameter $\lambda$ by using variational methods.

Further, the authors in [9], applying a recent critical point theorem of Ricceri [7], established the existence of at least one nontrivial solution for the problem

$$
\left\{\begin{array}{l}
-u^{\prime \prime}=[\lambda f(x, u)+g(u)] h\left(x, u^{\prime}\right) \quad \text { in }(0,1) \\
u(0)=u(1)=0
\end{array}\right.
$$


where $\lambda$ is a positive parameter, $f:[0,1] \times \mathbb{R} \rightarrow \mathbb{R}$ is an $L^{1}$-Carathéodory function, $g$ : $\mathbb{R} \rightarrow \mathbb{R}$ is a Lipschitz continuous function with $g(0)=0$, and $h:[0,1] \times \mathbb{R} \rightarrow[0,+\infty)$ is a bounded and continuous function with $m:=\inf _{(x, t) \in[0,1] \times \mathbb{R}} h(x, t)>0$.

In the present paper, first we obtain the existence of at least one solution for problem (1.2). It is worth noticing that, usually, to obtain the existence of one solution, asymptotic conditions both at zero and at infinity on the nonlinear term are requested, while here it is assumed only a unique algebraic condition (see $\left(\mathrm{A}_{4}\right)$ in Theorem 3.3). As a consequence, by combining with the classical Ambrosetti-Rabinowitz condition (see [10]), the existence of two solutions is obtained (see Theorem 4.1). Subsequently, an existence result of three nonnegative solutions is obtained combining two algebraic conditions which guarantee the existence of two local minima for the Euler-Lagrange functional and applying the mountain pass theorem as given by Pucci and Serrin (see [11]) to ensure the existence of the third critical point (see Theorem 4.5).

As an example, we state here the following special case of Theorem 4.5.

Theorem 1.1 Let $h: \mathbb{R} \rightarrow[0,+\infty)$ be a bounded and continuous function with $\bar{m}:=$ $\inf _{t \in \mathbb{R}} h(t)>0$ and $\bar{M}:=\sup _{t \in \mathbb{R}} h(t)$, and $g: \mathbb{R} \rightarrow \mathbb{R}$ be a nonnegative continuous function with $g(0) \neq 0$ such that

$$
\lim _{t \rightarrow 0^{+}} \frac{g(t)}{t}=+\infty, \quad \lim _{t \rightarrow+\infty} \frac{g(t)}{t}=0,
$$

and

$$
\int_{0}^{1} g(x) d x<\frac{\bar{m}}{16 \bar{M}} \int_{0}^{2} g(x) d x
$$

Then, for each

$$
\lambda \in] \frac{32}{\bar{m} \int_{0}^{2} g(x) d x}, \frac{2}{\bar{M} \int_{0}^{1} g(x) d x}[
$$

the problem

$$
\left\{\begin{array}{l}
-u^{\prime \prime}(x)=\lambda g(u) h\left(u^{\prime}\right), \quad x \in(0,1), \\
u(0)=u(1)=0
\end{array}\right.
$$

admits at least three positive classical solutions in $W_{0}^{1,2}([0,1])$.

Finally, we point out that Theorem 1.1 ensures a precise conclusion in the sense that a location of the parameter $\lambda$ in order to obtain at least three distinct solutions is also provided.

\section{Preliminaries}

For a given nonempty set $X$ and two functionals $\Phi, \Psi: X \rightarrow \mathbb{R}$, we define the following functions:

$$
\beta\left(r_{1}, r_{2}\right):=\inf _{v \in \Phi^{-1}(] r_{1}, r_{2}[)} \frac{\sup _{u \in \Phi^{-1}(] r_{1}, r_{2}[)} \Psi(u)-\Psi(v)}{r_{2}-\Phi(v)},
$$




$$
\rho_{2}\left(r_{1}, r_{2}\right):=\sup _{v \in \Phi^{-1}(] r_{1}, r_{2}[)} \frac{\Psi(v)-\sup _{\left.\left.u \in \Phi^{-1}(]-\infty, r_{1}\right]\right)} \Psi(u)}{\Phi(v)-r_{1}}
$$

for all $r_{1}, r_{2} \in \mathbb{R}$, with $r_{1}<r_{2}$, and

$$
\rho(r):=\sup _{v \in \Phi^{-1}([r,+\infty[)} \frac{\Psi(v)-\sup _{\left.\left.u \in \Phi^{-1}(]-\infty, r\right]\right)} \Psi(u)}{\Phi(v)-r}
$$

for all $r \in \mathbb{R}$.

Theorem 2.1 ([7]) Let X be a real Banach space; $\Phi: X \rightarrow \mathbb{R}$ be a sequentially weakly lower semicontinuous, coercive and continuously Gâteaux differentiable function whose Gâteaux derivative admits a continuous inverse on $X^{*} ; \Psi: X \rightarrow \mathbb{R}$ be a continuously Gâteaux differentiable function whose Gâteaux derivative is compact. Put $I_{\lambda}:=\Phi-\lambda \Psi$ and assume that there are $r_{1}, r_{2} \in \mathbb{R}$, with $r_{1}<r_{2}$, such that

$$
\beta\left(r_{1}, r_{2}\right)<\rho_{2}\left(r_{1}, r_{2}\right) .
$$

Then, for each $\lambda \in] \frac{1}{\rho_{2}\left(r_{1}, r_{2}\right)}, \frac{1}{\beta\left(r_{1}, r_{2}\right)}\left[\right.$, there is $u_{0, \lambda} \in \Phi^{-1}(] r_{1}, r_{2}[)$ such that $I_{\lambda}\left(u_{0, \lambda}\right) \leq I_{\lambda}(u)$ for all $u \in \Phi^{-1}(] r_{1}, r_{2}[)$ and $I_{\lambda}^{\prime}\left(u_{0, \lambda}\right)=0$.

Theorem 2.2 ([7]) Let $X$ be a real Banach space; $\Phi: X \rightarrow \mathbb{R}$ be a continuously Gâteaux differentiable function whose Gâteaux derivative admits a continuous inverse on $X^{*} ; \Psi$ : $X \rightarrow \mathbb{R}$ be a continuously Gâteaux differentiable function whose Gâteaux derivative is compact. Fix $\inf _{X} \Phi<r<\sup _{X} \Phi$ and assume that

$$
\rho(r)>0,
$$

and for each $\lambda>\frac{1}{\rho(r)}$ the function $I_{\lambda}=\Phi-\lambda \Psi$ is coercive.

Then, for each $\lambda>\frac{1}{\rho(r)}$, there is $u_{0, \lambda} \in \Phi^{-1}(] r,+\infty[)$ such that $I_{\lambda}\left(u_{0, \lambda}\right) \leq I_{\lambda}(u)$ for all $u \in$ $\Phi^{-1}(] r,+\infty[)$ and $I_{\lambda}^{\prime}\left(u_{0, \lambda}\right)=0$.

Let $h:[a, b] \times \mathbb{R} \rightarrow[0,+\infty)$ be a bounded and continuous function with

$$
m:=\inf _{(x, t) \in[a, b] \times \mathbb{R}} h(x, t)>0,
$$

and $f:[a, b] \times \mathbb{R} \rightarrow \mathbb{R}$ be an $L^{1}$-Carathéodory function.

We recall that $f:[a, b] \times \mathbb{R} \rightarrow \mathbb{R}$ is an $L^{1}$-Carathéodory function if

(a) $x \mapsto f(x, \xi)$ is measurable for every $\xi \in \mathbb{R}$;

(b) $\xi \mapsto f(x, \xi)$ is continuous for almost every $x \in[a, b]$;

(c) for every $\rho>0$, there is a function $l_{\rho} \in L^{1}([a, b])$ such that

$$
\sup _{|\xi| \leq \rho}|f(x, \xi)| \leq l_{\rho}(x)
$$

for almost every $x \in[a, b]$. 
Corresponding to $f$ and $h$, we introduce the functions $F:[a, b] \times \mathbb{R} \rightarrow \mathbb{R}$ and $H:[a, b] \times$ $\mathbb{R} \rightarrow[0,+\infty)$, respectively, as follows:

$$
F(x, t):=\int_{0}^{t} f(x, \xi) d \xi
$$

and

$$
H(x, t):=\int_{0}^{t}\left(\int_{0}^{\tau} \frac{(p-1)|\delta|^{p-2}}{h(x, \delta)} d \delta\right) d \tau
$$

for all $x \in[a, b]$ and $t \in \mathbb{R}$. Also, we use the following notation:

$$
M:=\sup _{(x, t) \in[a, b] \times \mathbb{R}} h(x, t) .
$$

Here and in the following, let $X:=W_{0}^{1, p}([a, b])$ equipped with the norm

$$
\|u\|:=\left(\int_{a}^{b}\left|u^{\prime}(x)\right|^{p} d x\right)^{1 / p}
$$

Then, $X$ is a reflexive real Banach space. Since $p>1, X$ is compactly embedded in $C^{0}([a, b])$ and

$$
\|u\|_{\infty} \leq \frac{(b-a)^{(p-1) / p}}{2}\|u\|
$$

for all $u \in X$ (see, e.g., [12]).

By a classical solution of problem (1.2), we mean a function $u$ such that $u \in C^{1}([a, b])$, $u^{\prime} \in A C([a, b])$, and $u(x)$ satisfies (1.2) a.e. on $[a, b]$. We say that a function $u \in X$ is a weak solution of problem (1.2) if

$$
\int_{a}^{b}\left(\int_{0}^{u^{\prime}(x)} \frac{(p-1)|\tau|^{p-2}}{h(x, \tau)} d \tau\right) v^{\prime}(x) d x-\lambda \int_{a}^{b} f(x, u(x)) v(x) d x=0
$$

for all $v \in X$.

The following lemma is taken from [6], Lemma 2.2.

Lemma 2.3 A weak solution to (1.2) in X coincides with a classical solution to (1.2).

We cite recent monographs [13-15] as general references for the basic notions used in the paper.

\section{Main results}

In this section we present our main results. To be precise, we establish an existence result of at least one solution, Theorem 3.1, which is based on Theorem 2.1, and we point out some consequences, Theorems 3.2, 3.3 and 3.4. Finally, we present another existence result of at least one solution, Theorem 3.7, which is based in turn on Theorem 2.2. 
Throughout the sequel, $\alpha, \beta$ are two positive constants such that $\alpha+\beta<b-a$. Now, put

$$
D:=\frac{(p-1)^{p-2}}{p}\left(\alpha^{-p+1}+\beta^{-p+1}\right) \text {. }
$$

Given two nonnegative constants $c, d$, with

$$
m(2 c)^{p} \neq D d^{p}(b-a)^{p-1} p M
$$

put

$$
a_{d}(c):=\frac{\int_{a}^{b} \max _{|t| \leq c} F(x, t) d x-\int_{a+\alpha}^{b-\beta} F(x, d) d x}{m(2 c)^{p}-D d^{p}(b-a)^{p-1} p M} .
$$

Theorem 3.1 Assume that there exist a nonnegative constant $c_{1}$ and two positive constants $c_{2}, d$, with

$$
c_{1}<\frac{(b-a)^{(p-1) / p}(p D)^{1 / p}}{2} d<\left(\frac{m}{M}\right)^{1 / p} c_{2}
$$

such that

$\left(\mathrm{A}_{1}\right) F(x, t) \geq 0$ for all $(x, t) \in([a, a+\alpha] \cup[b-\beta, b]) \times[0, d]$;

$\left(\mathrm{A}_{2}\right) a_{d}\left(c_{2}\right)<a_{d}\left(c_{1}\right)$.

Then, for each

$$
\left.\lambda \in \frac{1}{(b-a)^{p-1} p m M}\right] \frac{1}{a_{d}\left(c_{1}\right)}, \frac{1}{a_{d}\left(c_{2}\right)}[,
$$

problem (1.2) admits at least one nontrivial classical solution $\bar{u} \in X$ such that

$$
\frac{2 m^{1 / p}}{(b-a)^{(p-1) / p} M^{1 / p}} c_{1}<\|\bar{u}\|<\frac{2}{(b-a)^{(p-1) / p}} c_{2} .
$$

Proof Our aim is to apply Theorem 2.1 to our problem. To this end, for each $u \in X$, let the functionals $\Phi, \Psi: X \rightarrow \mathbb{R}$ be defined by

$$
\Phi(u):=\int_{a}^{b} H\left(x, u^{\prime}(x)\right) d x
$$

and

$$
\Psi(u):=\int_{a}^{b} F(x, u(x)) d x,
$$

and put

$$
I_{\lambda}(u):=\Phi(u)-\lambda \Psi(u) \quad \forall u \in X
$$

Note that the weak solutions of (1.2) are exactly the critical points of $I_{\lambda}$. The functionals $\Phi$ and $\Psi$ satisfy the regularity assumptions of Theorem 2.1. Indeed, by standard arguments, 
we have that $\Phi$ is continuously Gâteaux differentiable and its Gâteaux derivative is the functional $\Phi^{\prime}(u) \in X^{*}$ given by

$$
\Phi^{\prime}(u)(v)=\int_{a}^{b}\left(\int_{0}^{u^{\prime}(x)} \frac{(p-1)|\tau|^{p-2}}{h(x, \tau)} d \tau\right) v^{\prime}(x) d x
$$

for every $v \in X$. Since $\Phi^{\prime}$ is monotone (see the proof of [6], Lemma 2.1), by applying [16], Proposition 25.20, $\Phi$ is sequentially weakly lower semicontinuous. Again by [6], Lemma 2.1, $\Phi^{\prime}: X \rightarrow X^{*}$ admits a continuous inverse. On the other hand, the fact that $X$ is compactly embedded into $C^{0}([a, b])$ implies that the functional $\Psi$ is well defined, continuously Gâteaux differentiable and with compact derivative, whose Gâteaux derivative is given by

$$
\Psi^{\prime}(u)(v)=\int_{a}^{b} f(x, u(x)) v(x) d x
$$

for every $v \in X$.

Also, since $m \leq h(x, t) \leq M$ for all $(x, t) \in[a, b] \times \mathbb{R}$, we see that

$$
\frac{1}{p M}\|u\|^{p} \leq \Phi(u) \leq \frac{1}{p m}\|u\|^{p} \quad \text { for all } u \in X .
$$

Now, put

$$
r_{1}:=\frac{2^{p}}{(b-a)^{p-1} p M} c_{1}^{p}, \quad r_{2}:=\frac{2^{p}}{(b-a)^{p-1} p M} c_{2}^{p},
$$

and

$$
w(x):= \begin{cases}\frac{1}{\alpha^{p-1}} d(x-a)^{p-1} & \text { if } a \leq x<a+\alpha, \\ d & \text { if } a+\alpha \leq x \leq b-\beta, \\ \frac{1}{\beta^{p-1}} d(b-x)^{p-1} & \text { if } b-\beta<x \leq b .\end{cases}
$$

It is easy to verify that $w \in X$ and, in particular, one has

$$
\|w\|^{p}=d^{p}(p-1)^{p-2}\left(\alpha^{-p+1}+\beta^{-p+1}\right)=p D d^{p} .
$$

So, from (3.2), we have

$$
\frac{D d^{p}}{M} \leq \Phi(w) \leq \frac{D d^{p}}{m} .
$$

From condition (3.1), we obtain $r_{1}<\Phi(w)<r_{2}$. For all $u \in X$ such that $\Phi(u)<r_{2}$, taking (2.3) into account, one has $|u(x)|<c_{2}$ for all $x \in[a, b]$, from which it follows

$$
\begin{aligned}
\sup _{u \in \Phi^{-1}(]-\infty, r_{2}[)} \Psi(u) & =\sup _{u \in \Phi^{-1}(]-\infty, r_{2}[)} \int_{a}^{b} F(x, u(x)) d x \\
& \leq \int_{a}^{b} \max _{|t| \leq c_{2}} F(x, t) d x .
\end{aligned}
$$


Arguing as before, we obtain

$$
\sup _{\left.\left.u \in \Phi^{-1}(]-\infty, r_{1}\right]\right)} \Psi(u) \leq \int_{a}^{b} \max _{|t| \leq c_{1}} F(x, t) d x .
$$

Since $0 \leq w(x) \leq d$ for each $x \in[a, b]$, assumption $\left(\mathrm{A}_{1}\right)$ ensures that

$$
\Psi(w) \geq \int_{a+\alpha}^{b-\beta} F(x, d) d x
$$

Then we get

$$
\int_{a}^{b} \max _{|t| \leq c_{2}} F(x, t) d x \geq \int_{a+\alpha}^{b-\beta} F(x, d) d x
$$

and thus $a_{d}\left(c_{2}\right) \geq 0$. At this point, one has

$$
\begin{aligned}
\beta\left(r_{1}, r_{2}\right) & \leq \frac{\sup _{u \in \Phi^{-1}(]-\infty, r_{2}[)} \Psi(u)-\Psi(w)}{r_{2}-\Phi(w)} \\
& \leq \frac{\int_{a}^{b} \max _{|t| \leq c_{2}} F(x, t) d x-\int_{a+\alpha}^{b-\beta} F(x, d) d x}{\frac{2^{p}}{(b-a)^{p-1} p M} c_{2}^{p}-\frac{D d^{p}}{m}} \\
& =(b-a)^{p-1} p m M \frac{\int_{a}^{b} \max _{|t| \leq c_{2}} F(x, t) d x-\int_{a+\alpha}^{b-\beta} F(x, d) d x}{m\left(2 c_{2}\right)^{p}-D d^{p}(b-a)^{p-1} p M} \\
& =\left[(b-a)^{p-1} p m M\right] a_{d}\left(c_{2}\right) .
\end{aligned}
$$

Since $a_{d}\left(c_{2}\right) \geq 0$, hypothesis $\left(\mathrm{A}_{2}\right)$ implies that

$$
\int_{a}^{b} \max _{|t| \leq c_{1}} F(x, t) d x<\int_{a+\alpha}^{b-\beta} F(x, d) d x .
$$

So, one has

$$
\begin{aligned}
\rho_{2}\left(r_{1}, r_{2}\right) & \geq \frac{\Psi(w)-\sup _{\left.\left.u \in \Phi^{-1}(]-\infty, r_{1}\right]\right)} \Psi(u)}{\Phi(w)-r_{1}} \\
& \geq \frac{\int_{a+\alpha}^{b-\beta} F(x, d) d x-\int_{a}^{b} \max _{|t| \leq c 1} F(x, t) d x}{\frac{D d^{p}}{m}-\frac{2^{p}}{(b-a)^{p-1} p M} c_{1}^{p}} \\
& =(b-a)^{p-1} p m M \frac{\int_{a+\alpha}^{b-\beta} F(x, d) d x-\int_{a}^{b} \max _{|t| \leq c_{1}} F(x, t) d x}{D d^{p}(b-a)^{p-1} p M-m\left(2 c_{1}\right)^{p}} \\
& =\left[(b-a)^{p-1} p m M\right] a_{d}\left(c_{1}\right) .
\end{aligned}
$$

Hence, from assumption $\left(\mathrm{A}_{2}\right)$, one has $\beta\left(r_{1}, r_{2}\right)<\rho_{2}\left(r_{1}, r_{2}\right)$. Therefore, from Theorem 2.1, for each $\left.\lambda \in \frac{1}{(b-a)^{p-1} p m M}\right] \frac{1}{a_{d}\left(c_{1}\right)}, \frac{1}{a_{d}\left(c_{2}\right)}\left[\right.$, the functional $I_{\lambda}$ admits at least one critical point $\bar{u}$ such that

$$
r_{1}<\Phi(\bar{u})<r_{2}
$$


that is,

$$
\frac{2 m^{1 / p}}{(b-a)^{(p-1) / p} M^{1 / p}} c_{1}<\|\bar{u}\|<\frac{2}{(b-a)^{(p-1) / p}} c_{2} .
$$

So, applying Lemma 2.3 , the conclusion is achieved.

Now, we point out an immediate consequence of Theorem 3.1.

Theorem 3.2 Assume that there exist two positive constants $c, d$, with

$$
\frac{(b-a)^{(p-1) / p}(p D)^{1 / p}}{2} d<\left(\frac{m}{M}\right)^{1 / p} c
$$

such that assumption $\left(\mathrm{A}_{1}\right)$ in Theorem 3.1 holds. Furthermore, suppose that

$\left(\mathrm{A}_{3}\right)$

$$
\frac{\int_{a}^{b} \max _{|t| \leq c} F(x, t) d x}{m(2 c)^{p}}<\frac{\int_{a+\alpha}^{b-\beta} F(x, d) d x}{D d^{p}(b-a)^{p-1} p M} .
$$

Then, for each

$$
\lambda \in] \frac{D d^{p}}{m \int_{a+\alpha}^{b-\beta} F(x, d) d x}, \frac{(2 c)^{p}}{(b-a)^{p-1} p M \int_{a}^{b} \max _{|t| \leq c} F(x, t) d x}[,
$$

problem (1.2) admits at least one nontrivial classical solution $\bar{u} \in X$ such that $|\bar{u}(x)|<c$ for all $x \in[a, b]$.

Proof The conclusion follows from Theorem 3.1, by taking $c_{1}=0$ and $c_{2}=c$. Indeed, owing to assumption $\left(\mathrm{A}_{3}\right)$, one has

$$
\begin{aligned}
a_{d}(c) & =\frac{\int_{a}^{b} \max _{|t| \leq c} F(x, t) d x-\int_{a+\alpha}^{b-\beta} F(x, d) d x}{m(2 c)^{p}-D d^{p}(b-a)^{p-1} p M} \\
& <\frac{\left(1-\frac{D d^{p}(b-a)^{p-1} p M}{m(2 c)^{p}}\right) \int_{a}^{b} \max _{|t| \leq c} F(x, t) d x}{m(2 c)^{p}-D d^{p}(b-a)^{p-1} p M} \\
& =\frac{1}{m(2 c)^{p}} \int_{a}^{b} \max _{|t| \leq c} F(x, t) d x .
\end{aligned}
$$

On the other hand, one has

$$
a_{d}(0)=\frac{\int_{a+\alpha}^{b-\beta} F(x, d) d x}{D d^{p}(b-a)^{p-1} p M}
$$

Hence, taking assumption $\left(A_{3}\right)$ and (2.3) into account, Theorem 3.1 ensures the conclusion.

Now, we point out a special situation of our main result when the nonlinear term has separable variables. To be precise, let $\gamma \in L^{1}([a, b])$ such that $\gamma(x) \geq 0$ a.e. $x \in[a, b], \gamma \neq \equiv$, and let $g: \mathbb{R} \rightarrow \mathbb{R}$ be a nonnegative continuous function. Consider the following Dirichlet 
boundary value problem:

$$
\left\{\begin{array}{l}
-(p-1)\left|u^{\prime}(x)\right|^{p-2} u^{\prime \prime}(x)=\lambda \gamma(x) g(u) h\left(x, u^{\prime}\right), \quad x \in(a, b), \\
u(a)=u(b)=0 .
\end{array}\right.
$$

Put $G(t):=\int_{0}^{t} g(\xi) d \xi$ for all $t \in \mathbb{R}$, and set $\|\gamma\|_{1}:=\int_{a}^{b} \gamma(x) d x$.

Theorem 3.3 Assume that there exist two positive constants $c, d$, with

$$
\frac{(b-a)^{(p-1) / p}(p D)^{1 / p}}{2} d<\left(\frac{m}{M}\right)^{1 / p} c
$$

such that

$\left(\mathrm{A}_{4}\right)$

$$
\frac{G(c)}{c^{p}}<\left(\frac{2^{p} m \int_{a+\alpha}^{b-\beta} \gamma(x) d x}{D(b-a)^{p-1} p M\|\gamma\|_{1}}\right) \frac{G(d)}{d^{p}} .
$$

Then, for each

$$
\lambda \in] \frac{D}{m \int_{a+\alpha}^{b-\beta} \gamma(x) d x} \frac{d^{p}}{G(d)}, \frac{2^{p}}{(b-a)^{p-1} p M\|\gamma\|_{1}} \frac{c^{p}}{G(c)}[,
$$

problem (3.3) admits at least one positive classical solution $\bar{u} \in X$ such that $\bar{u}(x)<c$ for all $x \in[a, b]$.

Proof Put $f(x, \xi):=\gamma(x) g(\xi)$ for all $(x, \xi) \in[a, b] \times \mathbb{R}$. Clearly, one has $F(x, t)=\gamma(x) G(t)$ for all $(x, t) \in[a, b] \times \mathbb{R}$. Therefore, taking into account that $G$ is a nondecreasing function, Theorem 3.2 ensures the existence of a non-zero classical solution $\bar{u}$. We claim that it is nonnegative. In fact, arguing by contradiction and setting $A:=\{x \in[a, b]: \bar{u}(x)<0\}$, one has $A \neq \varnothing$. Put $\bar{v}:=\min \{\bar{u}, 0\}$, one has $\bar{v} \in X$. So, taking into account that $\bar{u}$ is a weak solution and by choosing $v=\bar{v}$, from our sign assumptions on the data, one has

$$
\int_{A}\left(\int_{0}^{\bar{u}^{\prime}(x)} \frac{(p-1)|\tau|^{p-2}}{h(x, \tau)} d \tau\right) \bar{u}^{\prime}(x) d x=\lambda \int_{A} \gamma(x) g(\bar{u}(x)) \bar{u}(x) d x \leq 0 .
$$

On the other hand,

$$
\frac{1}{M}\|\bar{u}\|_{W_{0}^{1, p}(A)}^{p} \leq \int_{A}\left(\int_{0}^{\bar{u}^{\prime}(x)} \frac{(p-1)|\tau|^{p-2}}{h(x, \tau)} d \tau\right) \bar{u}^{\prime}(x) d x
$$

So, $\|\bar{u}\|_{W_{0}^{1, p}(A)}=0$, which is absurd. Hence, our claim is proved. Now, owing to the strong maximum principle (see, e.g., [17], Theorem 11.1) the classical solution $\bar{u}$, being non-zero, is positive and the conclusion is achieved.

We now give a special case of our main result as follows.

Theorem 3.4 Assume that

(A $\left.\mathrm{A}_{5}\right) \lim _{t \rightarrow 0^{+}} \frac{g(t)}{t^{p-1}}=+\infty$. 
Furthermore, for each $c>0$, set

$$
\lambda_{c}^{\star}:=\frac{2^{p}}{(b-a)^{p-1} p M\|\gamma\|_{1}} \frac{c^{p}}{G(c)} .
$$

Then, for every $\lambda \in] 0, \lambda_{c}^{\star}[$, problem (3.3) admits at least one positive classical solution.

Proof Fix $c>0$ and $\lambda \in] 0, \lambda_{c}^{\star}\left[\right.$. From $\left(\mathrm{A}_{5}\right)$ we get $\lim _{t \rightarrow 0^{+}} \frac{t^{p}}{G(t)}=0$. Thus, there exists a positive constant $d$ satisfying

$$
d<\frac{2 m^{1 / p}}{(b-a)^{(p-1) / p}(p M D)^{1 / p}} c
$$

such that

$$
\frac{D}{m \int_{a+\alpha}^{b-\beta} \gamma(x) d x} \frac{d^{p}}{G(d)}<\lambda<\frac{2^{p}}{(b-a)^{p-1} p M\|\gamma\|_{1}} \frac{c^{p}}{G(c)} .
$$

Hence, owing to Theorem 3.3, for every $\lambda \in] 0, \lambda_{c}^{\star}[$, problem (3.3) admits at least one positive classical solution $\bar{u} \in X$. The proof is complete.

Remark 3.5 We claim that under the above assumptions, the mapping $\lambda \mapsto I_{\lambda}(\bar{u})$ is negative and strictly decreasing in $] 0, \lambda_{c}^{\star}$ [. Indeed, the restriction of the functional $I_{\lambda}$ to $\Phi^{-1}(] 0, r_{2}[)$, where $r_{2}:=\frac{\left(2 c_{2}\right)^{p}}{(b-a)^{p-1} p M}$, admits a global minimum, which is a critical point (local minimum) of $I_{\lambda}$ in $X$. Moreover, since $w \in \Phi^{-1}(] 0, r_{2}[)$ and

$$
\frac{\Phi(w)}{\Psi(w)} \leq \frac{D d^{p}}{m G(d) \int_{a+\alpha}^{b-\beta} \gamma(x) d x}<\lambda,
$$

we have

$$
I_{\lambda}(\bar{u}) \leq I_{\lambda}(w)=\Phi(w)-\lambda \Psi(w)<0 .
$$

Next, we observe that

$$
I_{\lambda}(u)=\lambda\left(\frac{\Phi(u)}{\lambda}-\Psi(u)\right)
$$

for every $u \in X$ and fix $0<\lambda_{1}<\lambda_{2}<\lambda_{c}^{\star}$. Set

$$
m_{\lambda_{1}}:=\left(\frac{\Phi\left(\bar{u}_{1}\right)}{\lambda_{1}}-\Psi\left(\bar{u}_{1}\right)\right)=\inf _{u \in \Phi^{-1}(] 0, r_{2}[)}\left(\frac{\Phi(u)}{\lambda_{1}}-\Psi(u)\right)
$$

and

$$
m_{\lambda_{2}}:=\left(\frac{\Phi\left(\bar{u}_{2}\right)}{\lambda_{2}}-\Psi\left(\bar{u}_{2}\right)\right)=\inf _{u \in \Phi^{-1}(] 0, r_{2}[)}\left(\frac{\Phi(u)}{\lambda_{2}}-\Psi(u)\right) .
$$

Clearly, as claimed before, $m_{\lambda_{i}}<0$ (for $i=1,2$ ), and $m_{\lambda_{2}} \leq m_{\lambda_{1}}$ thanks to $\lambda_{1}<\lambda_{2}$. Then the mapping $\lambda \mapsto I_{\lambda}(\bar{u})$ is strictly decreasing in $] 0, \lambda_{c}^{\star}[$ owing to

$$
I_{\lambda_{2}}\left(\bar{u}_{2}\right)=\lambda_{2} m_{\lambda_{2}} \leq \lambda_{2} m_{\lambda_{1}}<\lambda_{1} m_{\lambda_{1}}=I_{\lambda_{1}}\left(\bar{u}_{1}\right) .
$$

This concludes the proof of our claim. 
Remark 3.6 In other words, Theorem 3.4 ensures that if the asymptotic condition at zero $\left(A_{5}\right)$ is verified, then, for every parameter $\lambda$ belonging to the real interval $] 0, \lambda^{\star}$, where

$$
\lambda^{\star}:=\frac{2^{p}}{(b-a)^{p-1} p M\|\gamma\|_{1}} \sup _{c>0} \frac{c^{p}}{G(c)}
$$

problem (3.3) admits at least one positive classical solution $\bar{u} \in X$.

Finally, we present an application of Theorem 2.2 which we will use in the next section to obtain multiple solutions.

Theorem 3.7 Assume that there exist two constants $\bar{c}, \bar{d}$, with

$$
0<\frac{(b-a)^{(p-1) / p}(p D)^{1 / p}}{2} \bar{d}
$$

such that

(A6) $\int_{a}^{b} \max _{|t| \leq \bar{c}} F(x, t) d x<\int_{a+\alpha}^{b-\beta} F(x, \bar{d}) d x$;

$\left(\mathrm{A}_{7}\right) \lim \sup _{|t| \rightarrow+\infty} \frac{F(x, t)}{|t|^{p}} \leq 0$ uniformly in $x$.

Then, for each $\lambda>\tilde{\lambda}$, where

$$
\tilde{\lambda}:=\frac{D \bar{d}^{p}(b-a)^{p-1} p M-m(2 \bar{c})^{p}}{(b-a)^{p-1} p m M\left(\int_{a+\alpha}^{b-\beta} F(x, \bar{d}) d x-\int_{a}^{b} \max _{|t| \leq \bar{c}} F(x, t) d x\right)},
$$

problem (1.2) admits at least one nontrivial classical solution $\tilde{u} \in X$ such that

$$
\|\tilde{u}\|>\frac{2 m^{1 / p}}{(b-a)^{(p-1) / p} M^{1 / p}} \bar{c}
$$

Proof The functionals $\Phi$ and $\Psi$ defined in the proof of Theorem 3.1 satisfy all regularity assumptions requested in Theorem 2.2. Moreover, by standard computations, assumption $\left(\mathrm{A}_{7}\right)$ implies that $I_{\lambda}, \lambda>0$, is coercive. So, our aim is to verify condition (2.2) of Theorem 2.2. To this end, put

$$
r:=\frac{2^{p}}{(b-a)^{p-1} p M} \bar{c}^{p}
$$

and

$$
w(x):= \begin{cases}\frac{1}{\underline{\alpha}^{p-1}} \bar{d}(x-a)^{p-1} & \text { if } a \leq x<a+\alpha \\ \frac{1}{\beta^{p-1}} \bar{d}(b-x)^{p-1} & \text { if } a+\alpha \leq x \leq b-\beta, \beta<x \leq b .\end{cases}
$$

Arguing as in the proof of Theorem 3.1, we obtain that

$$
\rho(r) \geq(b-a)^{p-1} p m M \frac{\int_{a+\alpha}^{b-\beta} F(x, \bar{d}) d x-\int_{a}^{b} \max _{|t| \leq \bar{c}} F(x, t) d x}{D \bar{d}^{p}(b-a)^{p-1} p M-m(2 \bar{c})^{p}} .
$$

So, from our assumption it follows that $\rho(r)>0$. 
Hence, from Theorem 2.2 for each $\lambda>\tilde{\lambda}$, the functional $I_{\lambda}$ admits at least one local minimum $\tilde{u}$ such that

$$
\|\tilde{u}\|>\frac{2 m^{1 / p}}{(b-a)^{(p-1) / p} M^{1 / p}} \bar{c}
$$

and the conclusion is achieved.

\section{Multiplicity results}

The main aim of this section is to present multiplicity results. First, as a consequence of Theorem 3.1, taking into account the classical theorem of Ambrosetti and Rabinowitz, we have the following multiplicity result.

Theorem 4.1 Let the assumptions of Theorem 3.1 be satisfied, and $f(\cdot, 0) \neq 0$ in $(a, b)$. Moreover, let

$\left(\mathrm{A}_{8}\right)$ there exist positive constants $v$ and $R$ such that $v m>p M$, and for all $|t| \geq R$ and $x \in$ $[a, b]$, one has

$$
0<\nu F(x, t) \leq t \cdot f(x, t)
$$

Then, for each

$$
\left.\lambda \in \frac{1}{(b-a)^{p-1} p m M}\right] \frac{1}{a_{d}\left(c_{1}\right)}, \frac{1}{a_{d}\left(c_{2}\right)}[
$$

problem (1.2) admits at least two nontrivial classical solutions $\bar{u}_{1}, \bar{u}_{2}$ such that

$$
\frac{2 m^{1 / p}}{(b-a)^{(p-1) / p} M^{1 / p}} c_{1}<\left\|\bar{u}_{1}\right\|<\frac{2}{(b-a)^{(p-1) / p}} c_{2} .
$$

Proof Fix $\lambda$ as in the conclusion. So, Theorem 3.1 ensures that problem (1.2) admits at least one nontrivial classical solution $\bar{u}_{1}$ satisfying condition (4.1) which is a local minimum of the functional $I_{\lambda}$.

Now, we prove the existence of the second solution distinct from the first one. To this end, we must show that the functional $I_{\lambda}$ satisfies the hypotheses of the mountain pass theorem.

Clearly, the functional $I_{\lambda}$ is of class $C^{1}$ and $I_{\lambda}(0)=0$.

We can assume that $\bar{u}_{1}$ is a strict local minimum for $I_{\lambda}$ in $X$. Therefore, there is $\rho>0$ such that $\inf _{\left\|u-\bar{u}_{1}\right\|=\rho} I_{\lambda}(u)>I_{\lambda}\left(\bar{u}_{1}\right)$, so condition [18], $\left(I_{1}\right)$, Theorem 2.2, is verified.

From $\left(\mathrm{A}_{8}\right)$, by standard computations, there is a positive constant $C$ such that

$$
F(x, t) \geq C|t|^{v}
$$

for all $x \in[a, b]$ and $|t|>R$. In fact, setting $a(x):=\min _{|\xi|=R} F(x, \xi)$ and

$$
\varphi_{t}(s):=F(x, s t), \quad \forall s>0,
$$


by $\left(\mathrm{A}_{8}\right)$, for every $x \in[a, b]$ and $|t|>R$, one has

$$
0<\nu \varphi_{t}(s)=\nu F(x, s t) \leq s t \cdot f(x, s t)=s \varphi_{t}^{\prime}(s), \quad \forall s>\frac{R}{|t|} .
$$

Therefore,

$$
\int_{R /|t|}^{1} \frac{\varphi_{t}^{\prime}(s)}{\varphi_{t}(s)} d s \geq \int_{R /|t|}^{1} \frac{v}{s} d s
$$

Then

$$
\varphi_{t}(1) \geq \varphi_{t}\left(\frac{R}{|t|}\right) \frac{|t|^{\nu}}{R^{v}} .
$$

Taking into account (4.3), we obtain

$$
F(x, t) \geq F\left(x, \frac{R}{|t|} t\right) \frac{|t|^{\nu}}{R^{\nu}} \geq a(x) \frac{|t|^{\nu}}{R^{v}} \geq C|t|^{\nu},
$$

where $C>0$ is a constant. Thus, (4.2) is proved. Now, choosing any $u \in X \backslash\{0\}$, one has

$$
I_{\lambda}(t u)=(\Phi-\lambda \Psi)(t u) \leq \frac{t^{p}}{p m}\|u\|^{p}-\lambda t^{\nu} C \int_{a}^{b}|u(x)|^{\nu} d x \rightarrow-\infty
$$

as $t \rightarrow+\infty$ (since $v>p$ ). So, the functional $I_{\lambda}$ is unbounded from below and condition [18], $\left(I_{2}\right)$, Theorem 2.2, is verified. Therefore, $I_{\lambda}$ satisfies the geometry of mountain pass.

Now, to verify the Palais-Smale condition, it is sufficient to prove that any sequence of Palais-Smale is bounded. To this end, taking into account $\left(\mathrm{A}_{8}\right)$ one has

$$
\begin{aligned}
v I_{\lambda}\left(u_{n}\right)-\left\|I_{\lambda}^{\prime}\left(u_{n}\right)\right\|_{X^{*}}\left\|u_{n}\right\| \\
\quad \geq v I_{\lambda}\left(u_{n}\right)-I_{\lambda}^{\prime}\left(u_{n}\right)\left(u_{n}\right) \\
\quad=v \Phi\left(u_{n}\right)-\lambda \nu \Psi\left(u_{n}\right)-\Phi^{\prime}\left(u_{n}\right)\left(u_{n}\right)+\lambda \Psi^{\prime}\left(u_{n}\right)\left(u_{n}\right) \\
\quad \geq\left(\frac{v}{p M}-\frac{1}{m}\right)\left\|u_{n}\right\|^{p}-\lambda \int_{a}^{b}\left[\nu F\left(x, u_{n}(x)\right)-f\left(x, u_{n}(x)\right) u_{n}(x)\right] d x \\
\quad \geq\left(\frac{v}{p M}-\frac{1}{m}\right)\left\|u_{n}\right\|^{p} .
\end{aligned}
$$

If $\left\{u_{n}\right\}$ is not bounded, from (4.4) we have a contradiction. Thus, $I_{\lambda}$ satisfies the PalaisSmale condition.

Hence, the classical theorem of Ambrosetti and Rabinowitz ensures a critical point $\bar{u}_{2}$ of $I_{\lambda}$ such that $I_{\lambda}\left(\bar{u}_{2}\right)>I_{\lambda}\left(\bar{u}_{1}\right)$. So, $\bar{u}_{1}$ and $\bar{u}_{2}$ are two distinct classical solutions of (1.2) and the proof is complete.

Corollary 4.2 Assume that there exist two positive constants $c, d$, with

$$
\frac{(b-a)^{(p-1) / p}(p D)^{1 / p}}{2} d<\left(\frac{m}{M}\right)^{1 / p} c
$$

such that $\left(\mathrm{A}_{4}\right)$ holds. Assume also that 
$\left(\mathrm{A}_{9}\right)$ there exist positive constants $v$ and $R$ such that $\nu m>p M$, and for all $|t| \geq R$, one has

$$
0<v G(t) \leq t \cdot g(t)
$$

Then, for each

$$
\lambda \in] \frac{D}{m \int_{a+\alpha}^{b-\beta} \gamma(x) d x} \frac{d^{p}}{G(d)}, \frac{2^{p}}{(b-a)^{p-1} p M\|\gamma\|_{1}} \frac{c^{p}}{G(c)}[,
$$

problem (3.3) admits at least two nonnegative classical solutions $\bar{u}_{1}, \bar{u}_{2}$ such that $\bar{u}_{1}(x)<c$ for all $x \in[a, b]$.

Corollary 4.3 Assume that $\left(\mathrm{A}_{5}\right)$ and $\left(\mathrm{A}_{9}\right)$ are satisfied. Then, for each $\left.\lambda \in\right] 0, \lambda^{\star}[$, problem (3.3) admits at least two nonnegative classical solutions.

Next, as a consequence of Theorems 3.7 and 3.2, the following theorem of the existence of three classical solutions is obtained, and its consequence for the nonlinearity with separable variables is presented.

Theorem 4.4 Assume that $\left(\mathrm{A}_{7}\right)$ holds. Moreover, assume that there exist four positive constants $c, d, \bar{c}, \bar{d}$, with

$$
\frac{(b-a)^{(p-1) / p}(p D)^{1 / p}}{2} d<\left(\frac{m}{M}\right)^{1 / p} c \leq \bar{c}<\frac{(b-a)^{(p-1) / p}(p D)^{1 / p}}{2} \bar{d},
$$

such that $\left(\mathrm{A}_{3}\right),\left(\mathrm{A}_{6}\right)$ and

$\left(\mathrm{A}_{10}\right)$

$$
\frac{\int_{a}^{b} \max _{|t| \leq c} F(x, t) d x}{(2 c)^{p}}<\frac{m\left(\int_{a+\alpha}^{b-\beta} F(x, \bar{d}) d x-\int_{a}^{b} \max _{|t| \leq \bar{c}} F(x, t) d x\right)}{D \bar{d}^{p}(b-a)^{p-1} p M-m(2 \bar{c})^{p}}
$$

are satisfied. Then, for each

$$
\lambda \in \Lambda:=] \max \left\{\tilde{\lambda}, \frac{D d^{p}}{m \int_{a+\alpha}^{b-\beta} F(x, d) d x}\right\}, \frac{(2 c)^{p}}{(b-a)^{p-1} p M \int_{a}^{b} \max _{|t| \leq c} F(x, t) d x}[,
$$

problem (1.2) admits at least three classical solutions.

Proof First, we observe that $\Lambda \neq \varnothing$ owing to $\left(\mathrm{A}_{10}\right)$. Next, fix $\lambda \in \Lambda$. Theorem 3.2 ensures a nontrivial classical solution $\bar{u}$ such that

$$
\|\bar{u}\|<\frac{2}{(b-a)^{(p-1) / p}} c
$$

which is a local minimum for the associated functional $I_{\lambda}$, as well as Theorem 3.7 guarantees a nontrivial classical solution $\tilde{u}$ such that

$$
\|\tilde{u}\|>\frac{2 m^{1 / p}}{(b-a)^{(p-1) / p} M^{1 / p}} \bar{c}
$$

which is a local minimum for $I_{\lambda}$. Hence, the mountain pass theorem as given by Pucci and Serrin (see [11]) ensures the conclusion. 


\section{Theorem 4.5 Assume that}

$\left(A_{11}\right) \limsup \sup _{t \rightarrow 0^{+}} \frac{G(t)}{t^{p}}=+\infty$;

$\left(\mathrm{A}_{12}\right) \lim \sup _{t \rightarrow+\infty} \frac{G(t)}{t^{p}}=0$.

Further, assume that there exist two positive constants $\bar{c}, \bar{d}$, with

$$
\bar{c}<\frac{(b-a)^{(p-1) / p}(p D)^{1 / p}}{2} \bar{d},
$$

such that

$\left(\mathrm{A}_{13}\right)$

$$
\frac{G(\bar{c})}{\bar{c}^{p}}<\left(\frac{2^{p} m \int_{a+\alpha}^{b-\beta} \gamma(x) d x}{D(b-a)^{p-1} p M\|\gamma\|_{1}}\right) \frac{G(\bar{d})}{\bar{d}^{p}} .
$$

Then, for each

$$
\lambda \in] \frac{D}{m \int_{a+\alpha}^{b-\beta} \gamma(x) d x} \frac{\bar{d}^{p}}{G(\bar{d})}, \frac{2^{p}}{(b-a)^{p-1} p M\|\gamma\|_{1}} \frac{\bar{c}^{p}}{G(\bar{c})}[,
$$

problem (3.3) admits at least three nonnegative classical solutions.

Proof Clearly, $\left(\mathrm{A}_{12}\right)$ implies $\left(\mathrm{A}_{7}\right)$. Moreover, by choosing $d$ small enough and $c=\bar{c}$, simple computations show that $\left(A_{11}\right)$ implies $\left(A_{3}\right)$. Finally, from $\left(A_{13}\right)$ we get $\left(A_{6}\right)$ and, arguing as in the proof of Theorem 3.2, also $\left(\mathrm{A}_{10}\right)$. Hence, Theorem 4.4 ensures the conclusion.

Remark 4.6 If $g(0) \neq 0$, Corollaries 4.2 and 4.3 ensure two positive classical solutions while Theorem 4.5 ensures three positive classical solutions (see the proof of Theorem 3.3).

Finally, we present the following example to illustrate our results.

Example 4.7 Consider the following Dirichlet problem:

$$
\left\{\begin{array}{l}
-u^{\prime \prime}(x)=\lambda e^{x}\left(\frac{1}{6}+|u|^{2} u\right) h\left(u^{\prime}\right), \quad x \in(0,1), \\
u(0)=u(1)=0
\end{array}\right.
$$

where

$$
h(t):= \begin{cases}1 & \text { if } t<0 \\ \frac{6-t}{6} & \text { if } 0 \leq t \leq 1 \\ \frac{5}{6} & \text { if } t>1\end{cases}
$$

Then $h: \mathbb{R} \rightarrow[0,+\infty)$ is a bounded and continuous function with

$$
m=\inf _{t \in \mathbb{R}} h(t)=\frac{5}{6}>0 \quad \text { and } \quad M=\sup _{t \in \mathbb{R}} h(t)=1 .
$$

Let $g(t):=\frac{1}{6}+|t|^{2} t$ for all $t \in \mathbb{R}$. Obviously, $g(0) \neq 0$. Since

$$
\lim _{t \rightarrow 0^{+}} \frac{g(t)}{t}=\lim _{t \rightarrow 0^{+}}\left(\frac{1}{6 t}+|t|^{2}\right)=+\infty
$$


condition $\left(\mathrm{A}_{5}\right)$ holds true. Choose $v=3$ and $R=2$, we have $v m>p M$, and

$$
0<3 G(t) \leq t \cdot g(t)
$$

for all $|t| \geq 2$. Moreover, one has

$$
\lambda^{\star}=\frac{2}{\int_{0}^{1} e^{x} d x} \sup _{c>0} \frac{c^{2}}{G(c)}=\left(\frac{2}{e-1}\right) \sup _{c>0} \frac{12 c}{2+3 c^{3}} \geq\left.\left(\frac{2}{e-1}\right) \frac{12 c}{2+3 c^{3}}\right|_{c=\frac{1}{2}}=\frac{96}{19(e-1)} .
$$

Then, owing to Corollary 4.3 and Remark 4.6, for each $\lambda \in] 0, \frac{96}{19(e-1)}[$, problem (4.5) admits at least two positive classical solutions.

\section{Competing interests}

The authors declare that they have no competing interests.

\section{Authors' contributions}

All authors contributed equally to the writing of this paper. All authors read and approved the final manuscript.

\section{Author details}

${ }^{1}$ Department of Mathematics, Faculty of Mathematical Sciences, University of Mazandaran, Babolsar, Iran. ${ }^{2}$ Department of Mathematics, Faculty of Basic Sciences, University of Bojnord, P.O. Box 1339, Bojnord, 94531, Iran. ${ }^{3}$ Institute of Mathematics 'Simion Stoilow' of the Romanian Academy, P.O. Box 1-764, Bucharest, 014700, Romania. ${ }^{4}$ Topology, Geometry and Nonlinear Analysis Group, Department of Mathematics, University of Ljubljana, Jadranska 21, SI-1000, Ljubljana, Slovenia.

\section{Acknowledgements}

V Rădulescu acknowledges the support through Grant CNCS-PCE-47/2011.

Received: 17 April 2015 Accepted: 1 May 2015 Published online: 20 May 2015

\section{References}

1. Rădulescu, V: Finitely many solutions for a class of boundary value problems with superlinear convex nonlinearity. Arch. Math. (Basel) 84, 538-550 (2005)

2. Molica Bisci, G, Repovš, D: On sequences of solutions for discrete anisotropic equations. Expo. Math. 32, 284-295 (2014)

3. Agarwal, RP, Hong, H-L, Yeh, C-C: The existence of positive solutions for the Sturm-Liouville boundary value problems. Comput. Math. Appl. 35, 89-96 (1998)

4. Agarwal, RP, Bohner, M, Wong, PJ: Sturm-Liouville eigenvalue problems on time scales. Appl. Math. Comput. 99 153-166 (1999)

5. Agarwal, RP, O'Regan, D: An Introduction to Ordinary Differential Equations. Springer, New York (2008)

6. Graef, JR, Heidarkhani, S, Kong, L: A critical points approach for the existence of multiple solutions of a Dirichlet quasilinear system. J. Math. Anal. Appl. 388, 1268-1278 (2012)

7. Ricceri, B: A three critical points theorem revisited. Nonlinear Anal. 70, 3084-3089 (2009)

8. Afrouzi, GA, Hadjian, A: Infinitely many solutions for a class of Dirichlet quasilinear elliptic systems. J. Math. Anal. Appl. 393, 265-272 (2012)

9. Afrouzi, GA, Hadjian, A, Heidarkhani, S: Non-trivial solutions for a two-point boundary value problem. Ann. Pol. Math. 108, 75-84 (2013)

10. Ambrosetti, A, Rabinowitz, PH: Dual variational methods in critical point theory and applications. J. Funct. Anal. 14, 349-381 (1973)

11. Pucci, P, Serrin, J: A mountain pass theorem. J. Differ. Equ. 60, 142-149 (1985)

12. Talenti, G: Some inequalities of Sobolev type on two-dimensional spheres. In: Walter, W (ed.) General Inequalities, vol. 5. Internat. Ser. Numer. Math., vol. 80, pp. 401-408. Birkhäuser, Basel (1987)

13. Ciarlet, PG: Linear and Nonlinear Functional Analysis with Applications. SIAM, Philadelphia (2013)

14. Ghergu, M, Rădulescu, V: Singular Elliptic Problems: Bifurcation and Asymptotic Analysis. Oxford Lecture Ser. Math. Appl., vol. 37. Oxford University Press, New York (2008)

15. Kristály, A, Rădulescu, V, Varga, C: Variational Principles in Mathematical Physics, Geometry, and Economics: Qualitative Analysis of Nonlinear Equations and Unilateral Problems. Encyclopedia Math. Appl., vol. 136. Cambridge University Press, Cambridge (2010)

16. Zeidler, E: Nonlinear Functional Analysis and Its Applications, vols. II/B and III. Springer, Berlin (1985)

17. Pucci, P, Serrin, J: The strong maximum principle revisited. J. Differ. Equ. 196, 1-66 (2004); Erratum, ibid. 207, 226-227 (2004)

18. Rabinowitz, PH: Minimax Methods in Critical Point Theory with Applications to Differential Equations. CBMS Reg. Conf. Ser. Math., vol. 65. Am. Math. Soc., Providence (1986) 